\title{
Reduction in atherosclerotic events: a retrospective study in an outpatient cardiology practice
}

Anthony D. Mercando ${ }^{1}$, Hoang M. Lai², Wilbert S. Aronow ${ }^{2}$, Phoenix Kalen², Harit V. Desai², Kaushang Gandhi², Mala Sharma², Harshad Amin², Trung M. Lai

1Westchester Cardiology Associates/WestMed Medical Group and Department of Medicine, Columbia University College of Physicians and Surgeons, New York, USA

2Department of Medicine, New York Medical College, Valhalla, NY, USA

Submitted: 12 August 2011

Accepted: 7 October 2011

Arch Med Sci 2012; 8, 1: 57-62

DOI: 10.5114 /aoms.2012.27282

Copyright (c) 2012 Termedia \& Banach

\section{Abstract}

Introduction: Although atherosclerotic disease cannot be cured, risk of recurrent events can be reduced by application of evidence-based treatment protocols involving aspirin, beta blockers, angiotensin-converting enzyme inhibitors or angiotensin receptor blockers, and statin medications. We studied atherosclerotic event rates in a patient population treated before and after the development of aggressive risk factor reduction treatment protocols.

Material and methods: We performed a retrospective chart review of patients presenting for follow-up treatment of coronary artery disease in a community cardiology practice, comparing atherosclerotic event rates and medication usage in a 2-year treatment period prior to 2002 and a 2-year period in 2005-2008. Care was provided in both the early and later eras by 7 board-certified cardiologists in a suburban cardiology practice. Medication usage was compared in both treatment eras. The primary outcome was a composite event rate of myocardial infarction, cerebrovascular events, and coronary interventions.

Results: Three hundred and fifty-seven patients were studied, with a follow-up duration of $12.1( \pm 3.5)$ years. There were 132 composite events in 104 patients $(29.1 \%)$ in the early era compared to 40 events in 33 patients (9.2\%) in the later era $(p<0.0001)$. From the early to the later eras, there was an increase in use of $\beta$-blockers ( $66 \%$ to $83 \%, p<0.0001$ ), angiotensin-converting enzyme inhibitors or angiotensin receptor blockers (34\% to $80 \%, p<0.0001)$, and statins ( $40 \%$ to $90 \%, p<0.0001)$.

Conclusions: Application of aggressive evidence-based medication protocols for treatment of atherosclerosis is associated with a significant decrease in atherosclerotic events or need for coronary intervention.

Key words: atherosclerosis, myocardial infarction, cerebrovascular events, cardiovascular drugs.

\section{Introduction}

While mortality statistics still point to coronary heart disease as the number one killer in the United States [1], many cardiologists have perceived a dramatic change in this disease over the last 20 years, with reductions in incidence and severity noted by community physicians in clinical practice. In our practice of 7 cardiologists in a suburban New York City community, we noticed that the incidence of myocardial infarction and

\author{
Corresponding author: \\ Wilbert S. Aronow MD \\ Cardiology Division \\ New York Medical College \\ Macy Pavilion, Room 138 \\ Valhalla, New York, USA \\ Phone: (914) 493-5311 \\ Fax: (914) 235-6274 \\ E-mail: wsaronow@aol.com
}


need for percutaneous coronary intervention and coronary artery bypass surgery seemed to be decreasing in recent years compared with time periods prior to the late 1990s. Since use of statin medications and angiotensin-converting enzyme (ACE) inhibitors increased during the late 1990s and early 2000s, we hypothesized that the decrease in incidence of coronary heart disease events we were seeing was due to newer and more aggressive treatment regimens for outpatients, especially in secondary prevention. Multicenter studies have shown that aspirin, $\beta$-blockers, ACE inhibitors, and statin medications can be used to reduce the risk of coronary events. We decided to test our hypothesis in our own patient population by performing a retrospective review of patient charts to determine whether changes in medical therapy were associated with a decrease in the incidence of myocardial infarction and need for coronary interventions.

\section{Material and methods}

We examined paper and electronic chart records of 1770 randomly selected patients who presented to our outpatient cardiology practice in 2003-2006, and carried a diagnosis of coronary artery disease. We identified 357 patients in that group who initially presented with symptoms or signs of coronary artery disease before January 1, 2000 and were treated continuously through 2005-2008. For each patient, we looked at progress notes of all interim office visits and recorded medication usage, blood pressure, laboratory studies including lipid levels, and occurrence of adverse cardiovascular events from the time of initial presentation to last followup. Adverse events included occurrence of acute myocardial infarction or stroke, or need for coronary intervention, either percutaneous or surgical.

We compared the earliest 2-year periods for each patient during the first era, to the latest 2-year period of follow-up in the second era. A total of $1748 \mathrm{vi}$ sits in those 2 two-year periods were abstracted. If a patient presented initially with an adverse cardiovascular event, that event was not included as an event in the early era. For baseline data collec-

Table I. Characteristics of patients and study eras

\begin{tabular}{|lc|}
\hline Number of patients & 357 \\
\hline Age at first date of therapy [years] & $62.7 \pm 10.8$ \\
\hline Age at second era [years] & $74.7 \pm 10.6$ \\
\hline Men & $246(69 \%)$ \\
\hline Women & $111(31 \%)$ \\
\hline Duration of follow-up [years] & $12.1 \pm 3.5$ \\
\hline Year range for early era & $1977-2001$ \\
\hline Year range for later era & $2003-2008$ \\
\hline
\end{tabular}

tion, comorbidities of coronary artery disease, hyperlipidemia, diabetes mellitus, hypertension, congestive heart failure, angina, atrial fibrillation, carotid stenosis, transient ischemic attack, cerebrovascular accident, chronic kidney disease, cigarette smoking history, peripheral vascular disease, abdominal aortic aneurysm, and previous coronary event or intervention were recorded. Occurrence of subsequent myocardial infarction, transient ischemic attack, or cerebrovascular accident was recorded, as well as requirement for percutaneous coronary intervention or coronary artery bypass grafting. Dates of the events as well as dates of all medication initiation and discontinuation were recorded. Coronary artery disease was diagnosed as previously described [2-5]. Cerebrovascular accident was diagnosed as previously described [6-8].

The data were extracted from paper and electronic charts by the physician authors. Data collection was tabulated with Microsoft Access 2003, and statistical analysis was performed in Microsoft Excel 2003 (Microsoft Corporation, Redmond, WA, USA). Paired $t$-testing was used to analyze for statistical significance. A $p$-value of $<0.05$ was considered statistically significant.

\section{Results}

Demographics and associated clinical characteristics are shown in Tables I and II. The group consisted of 246 males (69\%) and 111 females. Average age at the time of the first visit was $62.7 \pm 10.8$ years and at the last visit was $74.7 \pm 10.6$ years. All patients had evidence for coronary artery disease at the time of presentation (angina, positive stress test, coronary artery disease on cardiac catheterization, or prior history of myocardial infarction, percutaneous coronary intervention ( $\mathrm{PCl})$, or coronary artery bypass surgery (CABS)). Other comorbidities included hyperlipidemia in 322 (90\%) of the patients, diabetes in 80 (22\%), hypertension in 295 (83\%), congestive heart failure in $58(16 \%)$, angina in $47(13 \%)$, and atrial fibrillation in 69 (19\%). One hundred and fifty-six (44\%) of the patients were smokers. Other comorbidities are shown in Table II. The primary endpoint of the study was a composite of myocardial infarction, cerebrovascular event (transient ischemic attack or stroke), or requirement for coronary intervention (either $\mathrm{PCl}$ or CABS).

One hundred and thirty-five (37.8\%) of the patients had myocardial infarction prior to (126 patients) or at their first visit, 67 (18.8\%) had PCl prior to (62 patients) or at their first visit, 59 (16.5\%) had CABS prior to (55 patients) or at their first visit, 4 patients had transient ischemic attack prior to (3 patients) or at their first visit, and 8 patients had history of cerebrovascular accident prior to their first visit. Only events or interventions that occurred 
after the first visit in the early era were counted as an event in that era. The early follow-up era consisted of 2-year periods which were completed before December 31, 2001 (range of start dates from March 3, 1977 to December 27, 1999). The later follow-up era consisted of 2 year periods which were completed before November 7, 2008 (range of start dates September 21, 2003 to November 6, 2006).

The composite endpoint of myocardial infarction, cerebrovascular event (transient ischemic attack or stroke), and requirement for coronary intervention was significantly reduced in the later follow-up era compared to the early follow-up era (Table III, Figure 1). The need for CABS and $\mathrm{PCI}$ decreased from the early era to the later era. Percutaneous coronary interventions decreased from 63 interventions in 50 patients (14\%) in the early era to 17 interventions in 16 patients (4.5\%) in the later era $(p<0.0001)$. Requirement for CABS decreased from 53 procedures in 53 patients (14.8\%) in the early era to 8 procedures in 8 patients $(2.2 \%)$ in the later era $(p<0.0001)$. Five patients of 50 who underwent $\mathrm{PCl}$ in the early era required $\mathrm{CABS}$ in the early era, and 2 required CABS in the later era. Three patients who had a $\mathrm{PCl}$ in the early era required a repeat $\mathrm{PCl}$ in the later era. Only 1 patient of 53 who had a CABS in the early era required a CABS in the later era. In the early era, 9 patients (2.5\%) experienced a total of 10 myocardial infarctions, while in the later era, 5 patients (1.4\%) experienced myocardial infarctions, a result that was not statistically significant. Incidences of transient ischemic attack and cerebrovascular accident were also statistically unchanged. Of note, 4 of the 5 patients (80\%) who experienced a myocardial infarction in the later era were not on statin medications.

Medication treatment regimens during these 2 follow-up periods changed significantly (Table IV). Statin use increased between the early era and the later era from 142 patients (39.8\%) to 323 patients (90.5\%, $p<0.0001)$. $\beta$-Blocker use increased from 235 patients $(65.8 \%)$ to 295 patients $(82.6 \%$, $p<0.0001)$. Angiotensin converting enzyme inhib-
Table II. Associated clinical characteristics and comorbidities of patients in the study

\begin{tabular}{|lc|}
\hline Comorbidity & $n(\%)$ \\
\hline Coronary artery disease & $357(100)$ \\
\hline Hyperlipidemia & $322(90)$ \\
\hline Diabetes mellitus & $80(22)$ \\
\hline Hypertension & $295(83)$ \\
\hline Clinical congestive heart failure & $58(16)$ \\
\hline Angina & $47(13)$ \\
\hline Atrial fibrillation & $69(19)$ \\
\hline Carotid stenosis & $19(5)$ \\
\hline Transient ischemic attack & $22(6)$ \\
\hline Cerebrovascular accident & $39(11)$ \\
\hline Chronic kidney disease & $10(3)$ \\
\hline Smoker at time of presentation & $156(44)$ \\
\hline Peripheral vascular disease & $40(11)$ \\
\hline Aortic abdominal aneurysm & $16(4)$ \\
\hline Prior myocardial infarction & $135(38)$ \\
\hline Prior percutaneous coronary intervention & $67(19)$ \\
\hline Prior coronary artery bypass surgery & $59(17)$ \\
\hline Prior transient ischemic attack & $4(1)$ \\
\hline Prior cerebrovascular accident & $8)$ \\
\hline
\end{tabular}

itor use increased from 101 patients in the early era $(28.3 \%)$ to 216 in the later era $(60.5 \%$, $p<0.0001)$. Use of angiotensin receptor blockers also increased from 25 patients (7.0\%) in the early era to $98(27.5 \%, p<0.0001)$ in the later.

Therefore, use of either ACE inhibitors or angiotensin receptor blockers increased from 34\% to $80 \%$. Although use of ezetimibe also increased from none in the early era to 100 patients (28.0\%) in the later era, all but 3 of these patients were placed on ezetimibe together with a statin medication. Aspirin and calcium channel blocker use did not change. Use of other agents also increased (clopidogrel, warfarin, insulin, thiazolidinediones,

Table III. Outcomes in the patient group in the early era vs. later era

\begin{tabular}{|lccccc|}
\hline Outcomes & \multicolumn{2}{c}{ Early era } & \multicolumn{2}{c}{ Later era } & \multirow{2}{*}{ Value of $p$} \\
\cline { 2 - 5 } & $\begin{array}{c}\text { Adverse } \\
\text { events, } n\end{array}$ & $\begin{array}{c}\text { Patients with } \\
\text { adverse events, } n(\%)\end{array}$ & $\begin{array}{c}\text { Adverse } \\
\text { events, } n\end{array}$ & $\begin{array}{c}\text { Patients with } \\
\text { adverse events, } n(\%)\end{array}$ & \\
\hline MI & 10 & $9(2.5)$ & 5 & $5(1.4)$ & 0.29 \\
\hline PCI & 63 & $50(14.0)$ & 17 & $16(4.5)$ & $<0.001$ \\
\hline CABS & 53 & $53(14.8)$ & 8 & $8(2.2)$ & $<0.001$ \\
\hline TIA & 2 & $2(0.6)$ & 1 & $1(0.3)$ & 0.56 \\
\hline CVA & 4 & $4(1.1)$ & 9 & $8(2.2)$ & 0.25 \\
\hline Composite & 132 & $104(29.1)$ & 40 & $33(9.2)$ & $<0.001$ \\
\hline
\end{tabular}

$\mathrm{MI}$ - myocardial infarction, $\mathrm{PCl}$ - percutaneous coronary intervention, CABS - coronary artery bypass surgery, TIA - transient ischemic attack, CVA - cerebrovascular accident 


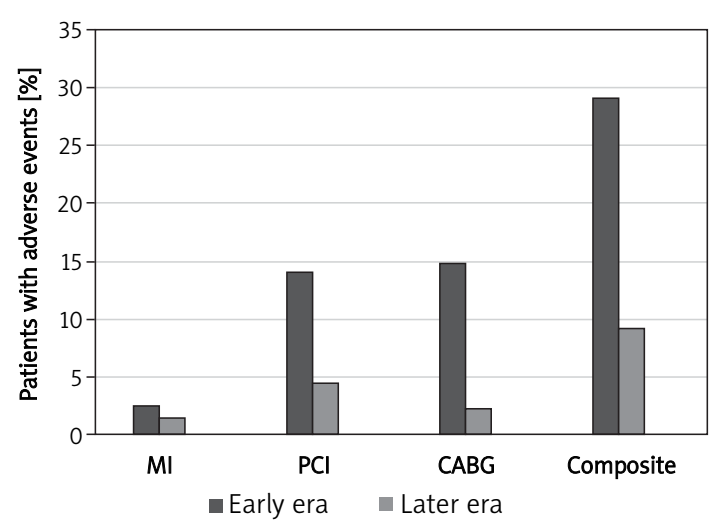

Figure 1. Percent of patients with events in early era and later era

$\mathrm{MI}$ - myocardial infarction, $\mathrm{PCl}$ - percutaneous coronary intervention, $C A B G$ - coronary artery bypass grafting

Table IV. Medication use for at least one visit in the earlier and later eras

\begin{tabular}{|c|c|c|c|}
\hline Medications & $\begin{array}{l}\text { Earlier era } \\
\quad n(\%)\end{array}$ & $\begin{array}{l}\text { Later era } \\
n(\%)\end{array}$ & Value of $p$ \\
\hline Statins & $142(40)$ & $323(90)$ & $<0.001$ \\
\hline Ezetimibe & $0(0)$ & $100(28)$ & $<0.001$ \\
\hline Nicotinic acid & $11(3)$ & $13(4)$ & 0.67 \\
\hline $\begin{array}{l}\text { Bile acid } \\
\text { sequestrants }\end{array}$ & $2(1)$ & $1(0.3)$ & 0.56 \\
\hline Fibrates & $17(5)$ & $18(5)$ & 0.84 \\
\hline Fish oils & $2(1)$ & $22(6)$ & $<0.001$ \\
\hline$\beta$-Blockers & $235(66)$ & $295(83)$ & $<0.001$ \\
\hline Diuretics & $115(32)$ & $202(57)$ & $<0.001$ \\
\hline ACE-I & $101(28)$ & $216(61)$ & $<0.001$ \\
\hline ARB & $25(7)$ & $98(27)$ & $<0.001$ \\
\hline ACE-I or ARB & $123(34)$ & $285(80)$ & $<0.001$ \\
\hline $\begin{array}{l}\text { Calcium channel } \\
\text { blocker }\end{array}$ & $161(45)$ & $148(41)$ & 0.24 \\
\hline Aspirin & $278(78)$ & $282(79)$ & 0.67 \\
\hline Ticlopidine & $17(5)$ & $0(0)$ & $<0.001$ \\
\hline Clopidogrel & $18(5)$ & $62(17)$ & $<0.001$ \\
\hline $\begin{array}{l}\text { Aspirin/ } \\
\text { extended-release } \\
\text { dipyridamole }\end{array}$ & $1(0.3)$ & $7(2)$ & 0.03 \\
\hline Warfarin & $50(14)$ & $95(27)$ & $<0.001$ \\
\hline Nitrates & $108(30)$ & $64(18)$ & $<0.001$ \\
\hline Digoxin & $56(16)$ & 64 (18) & 0.29 \\
\hline Cilostazol & $0(0)$ & $6(2)$ & 0.01 \\
\hline Insulin & $15(4)$ & $30(8)$ & $<0.001$ \\
\hline Thiazolidinediones & $8(2)$ & $38(11)$ & $<0.001$ \\
\hline Sulfonylureas & $32(9)$ & 49 (14) & 0.006 \\
\hline Metformin & $20(6)$ & $48(13)$ & $<0.001$ \\
\hline Sitagliptin & $0(0)$ & $2(1)$ & 0.16 \\
\hline
\end{tabular}

$A C E-I$ - angiotensin converting enzyme inhibitors, $A R B$ - angiotensin receptor blockers and metformin), but these drugs were used in only a small percentage of the patients in both eras. Clopidogrel was used with aspirin in all but 2 patients in the early era, and in all but 8 patients in the later era. Use of long-acting nitrates declined from $30 \%$ to $18 \%$.

\section{Discussion}

Coronary atherosclerosis is still a major cause of morbidity and mortality in the United States population, but our data shows that with aggressive treatment using evidence-based protocols, even patients with overt coronary artery disease can reduce their chance of developing subsequent events or requirement for interventions. Peroxisome proliferator-activated receptor-gamma and retinoid $X$ receptor- $\alpha$ expression down-regulation in both macrophages and smooth muscle cells was associated with more pronounced disease progression in patients with advanced carotid atherosclerotic lesions [9]. Further studies need to be performed to investigate the value of modulating vitamin $D$ signaling [10], using serotonin reuptake inhibitor antidepressants [11], and postprandial use of verjuice [12] in treatment of atherosclerosis.

The patients in this trial acted as their own controls, and although the group had a high prevalence of angina, myocardial infarction, positive stress tests, and need for coronary intervention prior to the year 2002, their chance of requiring coronary intervention decreased in later years, and incidence of myocardial infarction did not increase. Since coronary atherosclerosis is a progressive disease process, one would expect the incidence of events to increase over time; the opposite happened to the patients in this study, and the most likely reason is use of more aggressive preventive treatment protocols. This study shows that such aggressive therapies can be implemented successfully in a community cardiology practice. For the most part, these protocols involve medications such as statins, ACE inhibitors (and to a lesser extent angiotensin receptor blockers), aspirin, and $\beta$-blockers. While the use of aspirin did not increase significantly, there was a high rate of use of aspirin in both groups, and the use of clopidogrel increased significantly during this time.

Numerous studies have shown the benefits of statin drugs [13-15], ACE inhibitor [16-18], $\beta$-blockers [19-21], and aspirin [22, 23] for reducing events in patients with documented coronary artery disease, but there are little data examining the application of these evidence-based protocols in community clinical practice and over long periods of time. A randomized, blinded trial to investigate the application of these protocols in community practice is not possible for ethical reasons. Although this study is a retrospective analysis, it provides pre- 
sumptive data that changes in the practice of treating coronary disease over the past 20 years have significantly reduced event rates and the need for coronary interventions in this population.

Other possible contributing factors to the reduction in events include improved coronary intervention (CABS and $\mathrm{PCI}$ ) and lifestyle changes. However, even patients without interventions in the early era had no increase in the need for interventions in the later era. It is also unlikely that improved interventional techniques resulted in reduced need for repeat procedures, since only 6 patients who had $\mathrm{PCl}$ or $\mathrm{CABS}$ in the first era required a second procedure in the later era. While the physicians in our practice recommend lifestyle changes strongly to our patients, it is our experience that many patients do not follow these changes, and that many who do cannot maintain them over long periods of time; unfortunately, compliance with lifestyle changes is difficult to measure and quantify.

There are limitations to a retrospective study such as this one. Patients were not randomized to treatment protocols, but this study more closely mimics actual clinical treatment among community-based cardiologists. Adherence to medication treatment could not be determined, since techniques such as pill counts were not employed, but it is our experience that our patient population tends to follow medication regimens. Mortality differences were not examined in this study since the criteria for entry was attendance at the later era visit. It is possible that very sick patients who were seen in the early era could have died before they would have presented for follow-up in the later era. A part of benefit, possibly small, should be attributed to this factor.

This retrospective study should give community practitioners, both specialists and primary care providers, the encouragement to pursue aggressive cardiovascular risk reduction strategies as a means for reducing the need for cardiovascular procedures in their patients. More importantly, it provides patients with the knowledge that, while coronary artery disease cannot be cured, there is hope that with proper and aggressive risk factor reduction therapies it can be controlled.

\section{Acknowledgments}

The authors wish to thank the members of Westchester Cardiology Associates in Scarsdale, New York who do not appear as authors in this paper: Stanley Epstein MD, Mitchell Fishbach MD, Gary Gabelman MD, Richard Grose MD, Douglas Hart MD, and Gabriela Grasa MD. Their superb care provided the benefits to their patients cited in this paper.

None of the authors have any conflicts of interest. The study was performed without any outside financial support.

\section{References}

1. Centers for Disease Control and Prevention, http://www. cdc.gov/nchs/fastats/deaths.htm. Accessed November 30, 2010.

2. Lai HM, Aronow WS, Rachdev A, et al. Incidence of mortality in 1,040 patients with coronary heart disease or hypertensive heart disease with normal and abnormal left ventricular ejection fraction and with normal and abnormal QRS duration. Arch Med Sci 2008; 4: 140-2.

3. Ramdeen N, Aronow WS, Chugh S, Asija A. Patients undergoing coronary angiography because of chest pain with hepatitis $C$ virus have a higher prevalence of obstructive coronary artery disease than a control group. Arch Med Sci 2008; 4: 452-4.

4. Kannam H, Aronow WS, Chilappa K, et al. Association of the $\mathrm{QRS}$ duration on the resting electrocardiogram with the severity of coronary artery disease in 2,196 patients undergoing coronary angiography for suspected coronary artery disease. Arch Med Sci 2009; 5: 163-5.

5. Shao JH, Aronow WS, Ravipati G, et al. Prevalence of a minimal luminal cross sectional area of coronary arteries $<4 \mathrm{~mm}^{2}$ determined by intravascular ultrasound in patients with coronary artery calcium scores of 0-100, 100-200, 200-300, 300-400, and >400 determined by cardiac computer tomography. Arch Med Sci 2009; 5: 172-4.

6. Lleva P, Aronow WS, Amin H, et al. Prevalence of electrocardiographic abnormalities in patients with ischemic stroke, intracerebral hemorrhage, and subarachnoid hemorrhage. Arch Med Sci 2008; 4: 259-62.

7. Ravipati G, Aronow WS, Kumbar S, et al. Patients with diabetes mellitus with ischemic stroke have a higher hemoglobin Alc level and a higher serum low-density lipoprotein cholesterol level than diabetics without ischemic stroke. Arch Med Sci 2009; 5: 391-3.

8. Amin $H$, Aronow WS, Lleva P, et al. Prevalence of transthoracic echocardiographic abnormalities in patients with ischemic stroke, intracerebral hemorrhage, and subarachnoid hemorrhage. Arch Med Sci 2010; 6: 40-2.

9. Giaginis C, Klonaris C, Katsargyris A, et al. Correlation of peroxisome proliferator-activated receptor-gamma (PPARgamma) and retinoid $X$ receptor-alpha (RXR-alpha) expression with clinical risk factors in patients with advanced carotid atherosclerosis. Med Sci Monit 2011; 17: CR381-91.

10. Hu P, Hu B, Wang J, et al. Modulation of vitamin D signaling is a potential therapeutic target to lower cardiovascular risk in chronic kidney disease. Med Sci Monit 2011; 17: HY14-20.

11. Wozniak G, Toska A, Saridi M, Mouzas O. Seotonin reuptake inhibitor antidepressants (SSRIs) against atherosclerosis. Med Sci Monit 2011; 17: RA205-14.

12. Setorki M, Asgary S, Eidi A, Haeri Rohani A. Effects of acute verjuice consumption with a high-cholesterol diet on some biochemical risk factors of atherosclerosis in rabbits. Med Sci Monit 2010; 16: BR124-30.

13. Cannon CP, Braunwald E, McCabe $\mathrm{CH}$, et al.; Pravastatin or Atorvastatin Evaluation and Infection TherapyThrombolysis in Myocardial Infarction 22 Investigators. Intensive versus moderate lipid lowering with statins after acute coronary syndromes. N Engl J Med 2004; 350: 1495-504.

14. Heart Protection Study Collaborative Group. MRC/BHF Heart Protection Study of cholesterol lowering with simvastatin in 20,536 high-risk individuals: a randomised placebo-controlled trial. Lancet 2002; 360: 7-22. 
15. Shepherd J, Blauw GJ, Murphy MB, et al. PROspective Study of Pravastatin in the Elderly at Risk. Pravastatin in elderly individuals at risk of vascular disease (PROSPER): a randomised controlled trial. Lancet 2002; 360: 1623-30.

16. Yusuf S, Sleight P, Pogue J, Bosch J, Davies R, Dagenais G. Effects of an angiotensin-converting-enzyme inhibitor, ramipril, on cardiovascular events in high-risk patients. The Heart Outcomes Prevention Evaluation Study Investigators. N Engl J Med 2000; 342: 145-53.

17. Fox KM; EURopean trial On reduction of cardiac events with Perindopril in stable coronary Artery disease Investigators. Efficacy of perindopril in reduction of cardiovascular events among patients with stable coronary artery disease: randomised, double-blind, placebo-controlled, multicentre trial (the EUROPA study). Lancet 2003; 362: 782-8.

18. Braunwald E, Domanski MJ, Fowler SE, et al. Angiotensinconverting-enzyme inhibition in stable coronary artery disease. N Engl J Med 2004; 351: 2058-68.

19. Beta-Blocker Heart Attack Study Group. The beta-blocker heart attack trial. JAMA 1981; 246: 2073-4.

20. Gottlieb SS, McCarter RJ, Vogel RA. Effect of beta-blockade on mortality among high-risk and low-risk patients after myocardial infarction. N Engl J Med 1998; 339: 489-97.

21. Bielecka-Dabrowa A, Aronow WS, Rysz J, Banach M. Current place of beta-blockers in the treatment of hypertension. Curr Vasc Pharmacol 2010; 8: 733-41.

22. Baigent C, Blackwell L, Collins R, et al.; Antithrombotic Trialists' (ATT) Collaboration. Aspirin in the primary and secondary prevention of vascular disease: collaborative meta-analysis of individual participant data from randomised trials. Lancet 2009; 373: 1849-60.

23. US Preventive Services Task Force. Aspirin for the prevention of cardiovascular disease: U.S. Preventive Services Task Force recommendation statement. Ann Intern Med 2009; 150: 396-404. 